\title{
Larval density mediates knockdown resistance to pyrethroid insecticides in adult Aedes aegypti
}

\author{
Marissa K. Grossman ${ }^{1 *}$, Valentin Uc-Puc ${ }^{2}$, Adriana E. Flores ${ }^{3}$, Pablo C. Manrique-Saide ${ }^{2}$ \\ and Gonzalo M. Vazquez-Prokopec ${ }^{4}$
}

\begin{abstract}
Background: Understanding mechanisms driving insecticide resistance in vector populations remains a public health priority. To date, most research has focused on the genetic mechanisms underpinning resistance, yet it is unclear what role environmental drivers may play in shaping phenotypic expression. One of the key environmental drivers of Aedes aegypti mosquito population dynamics is resource-driven intraspecific competition at the larval stage. We experimentally investigated the role of density-dependent larval competition in mediating resistance evolution in Ae. aegypti, using knockdown resistance $(k d r)$ as a marker of genotypic resistance and CDC bottle bioassays to determine phenotype. We reared first-instar larvae from susceptible and pyrethroid-resistant field-derived populations of Ae. aegypti at high and low density and measured the resulting phenotypic resistance and population $k d r$ allele frequencies.

Results: At low density, only $48.2 \%$ of the resistant population was knocked down, yet at high density, the population was no longer phenotypically resistant - 93\% were knocked down when exposed to permethrin, which is considered susceptible according to WHO guidelines. Furthermore, the frequency of the C1534 kdr allele in the resistant population at high density decreased from $0.98 \pm 0.04$ to $0.69 \pm 0.04$ in only one generation of selection.

Conclusions: Our results indicate that larval conditions, specifically density, can impact both phenotype and genotype of pyrethroid-resistant populations. Furthermore, phenotypic susceptibility to pyrethroids may be re-established in a resistant population through a gene $x$ environment interaction, a finding that can lead to the development of novel resistance management strategies that capitalize on density-induced costs.
\end{abstract}

Keywords: Pyrethroid resistance, Aedes aegypti, Intraspecific competition, $k d r$

\section{Background}

Insecticide resistance poses a significant threat to the control of both agricultural pests and vectors of human disease. As insecticides remain one of the pillars of contemporary vector-borne disease control, curtailing resistance evolution or delaying undesirable impacts of resistance on pathogen transmission are global health priorities [1]. Current research on insecticide resistance in disease vectors has mainly focused on its evolutionary underpinnings: the genes responsible, the physiologic pathways involved, and how different control methods

\footnotetext{
* Correspondence: mzg175@psu.edu

'Department of Entomology, Pennsylvania State University, University Park, Pennsylvania, PA 16802, USA

Full list of author information is available at the end of the article
}

can potentially delay the evolution of resistance [1-4]. While genetic mechanisms have been largely identified, it is still unclear what role environmental drivers play in shaping phenotypic expression. For example, it has been shown that rearing Anopheles spp. under reduced diet regimes significantly decreases their phenotypic resistance to permethrin [5] and DDT [6], and adult insecticide exposure at lower temperatures increases their phenotypic resistance to malathion [7]. Understanding the potential for gene $\times$ environment interactions to impact phenotype could lead to novel approaches for resistance management and mitigation.

Using Aedes aegypti mosquitoes as a study system, we aim to explore the impact of intraspecific competition at the larval stage on the genotype and phenotypic 
expression of pyrethroid resistance. Aedes aegypti, the main vector for dengue, chikungunya and Zika, experiences strong insecticide selection pressure from vector control efforts that are currently the only way to prevent disease outbreaks. Most Ae. aegypti control programs throughout the world employ ultra-low volume spraying (ULV), indoor residual spraying (IRS), and the application of larvicides to target individuals in both the aquatic and terrestrial life stages $[8,9]$. Consequently, $A e$. aegypti has developed resistance to every class of insecticide, with the most widespread being pyrethroid resistance [10]. The most common mechanism conferring resistance to synthetic neurotoxins such as pyrethroids is called "knockdown resistance" or $k d r$. These are point mutations in the para-orthologous sodium channel gene that alter the ability of the insecticide to bind to the voltage-gated sodium channels in the mosquito's nerve cell membranes [11]. Given the critical role of voltagegated sodium channels in nervous system functioning, the presence of $k d r$ mutations within a mosquito's genome has important pleiotropic effects on mosquito behavior, performance and overall fitness [12-14].

It has been well established that density-dependent growth at the larval stage is one of the main exogenous factors shaping Ae. aegypti population dynamics [15-18]. In natural conditions, the Ae. aegypti life-cycle, which involves four free-living aquatic larval stages and a pupal stage that does not feed, occurs mainly in man-made containers such as buckets or flower pots [19]. During the larval period, strong indirect competition for resources occurs, particularly in larval habitats with limited food availability $[15,16,20]$. At high larval density, per-capita consumption is reduced, leading to smaller adult mosquitoes, slower development time, and decreased adult survival [15-17]. What is yet unknown is whether intraspecific competition in the larval stage can have a differential impact on the performance of insecticideresistant or susceptible individuals, thereby influencing resistance evolution. For example, Raymond et al. [21] found that under high density conditions, a Cry1Ac (Bacillus thuringiensis toxin) resistant population of diamondback moth (Plutella xylostella) had reduced survival compared to the susceptible population, and the resistant population also experienced a significant decline in phenotypic resistance in only three generations.

The strong bottom-up population regulation exerted by larval intraspecific competition in Ae. aegypti led us to hypothesize that density-dependent competition can significantly influence the allelic frequency of the $k d r$ mutations in a population and influence the rates of resistance evolution. To test this hypothesis, we designed a series of larval competition experiments to investigate the role of densitydependence at the larval stage on the rates of genotypic and phenotypic resistance to pyrethroid insecticides.

\section{Methods}

\section{Experimental design}

To quantify the impact of larval intraspecific competition on the resulting insecticide resistance status, we created a fully factorial experiment with two factors: density and population. There were two levels of density, low (50 larvae) and high (500 larvae), which represent the lower and upper range of larval density described in Merida, Mexico during the dengue transmission season $[22,23]$. We used F1 larvae from two Ae. aegypti field populations: susceptible ( $10 \%$ frequency of both $k d r$ mutations and 100\% knockdown to permethrin at the diagnostic time) and resistant (98\% frequency of the C1534 mutation, $73 \%$ of the I1016 mutation and 13\% knockdown to permethrin at the diagnostic time). We then mixed the two populations as first-instar larvae (see below for details) to create a third, intermediate resistance level. Our experimental design thus involved six treatment combinations (2 densities and 3 resistance levels), which were replicated five times each.

We used 21 white experimental buckets, which is the typical size habitat for Ae. aegypti in Merida, and filled each one with 11 of municipal water [23]. We placed either 50 (low density treatment) or 500 (high density treatment) first-instar larvae from each population (susceptible or resistant) into an experimental bucket. The intermediate resistance level was created by placing 25 or 250 firstinstar larvae from each resistance population into the same experimental bucket. Larvae were fed $50 \mathrm{mg}$ of bovine liver powder (MP Biomedicals LLC, Santa Ana, USA) every other day until all reached pupation. Buckets were covered with a mesh net to protect from oviposition of ambient mosquitoes and entrance of other organisms. The number of pupae and recently emerged adults in each bucket were counted daily. Adults were removed daily with a mouth aspirator and placed in an experimental cage (BugDorm-1 Insect Rearing Cage, MegaView Science, Talchung, Taiwan) and given $75-100 \mathrm{ml}$ of $5-10 \%$ sugar solution every day for hydration and nourishment.

Once all adult mosquitoes emerged in the low density treatment, 15 females and 15 males from each replicate were selected at random, euthanized by freezing, and stored individually in 100\% ethanol for further genotyping analysis. For the high density treatment, the same procedures were conducted; however, half of the 30 mosquitoes ( 7 males and 7 females) were removed halfway through the experiment (day 18), and the other half were removed at the end. Obtaining two samples over the duration of the high density experiment minimized any potential bias if emergence time differed between susceptible and resistant individuals.

Experiments were conducted in Merida, Mexico during February-May 2016, inside an urban residence to recreate the typical environmental fluctuations experienced by $A e$. 
aegypti populations. Temperature ranged from 21.9 to 37 . $1{ }^{\circ} \mathrm{C}$ and humidity from 40 to $82 \%$ throughout the course of the experiment, measured inside the experimental room with a RadioShack Indoor/Outdoor Thermometer (RadioShack, Fort Worth, USA).

\section{Phenotypic resistance assay}

Standardized Centers for Disease Control and Prevention (CDC) bottle bioassays were conducted on mosquitoes from each treatment replicate to determine phenotypic resistance. Four replicates of 25 mosquitoes each were placed in bottles coated with $15 \mu \mathrm{g} / \mathrm{ml}$ of technical grade permethrin according to CDC guidelines [24]. In the high density treatment, two of the four replicates were conducted at day 18, using mosquitoes that had emerged prior to that date (3-4 days-old), so as to not bias results as previously stated; the other two replicates were completed at the end of the experiment, which was between days 33-40 depending on the replicate. The low density treatment did not contain enough mosquitoes for the bottle bioassays since they only contained 50 mosquitoes at maximum, so two extra replicate buckets were simultaneously run but only used to complete the bioassays. The number of individuals knocked down was recorded every 10 minutes until all individuals were knocked down, but no longer than 120 minutes. The percentage of individuals knocked down at the diagnostic time of 30 minutes was calculated for each replicate, and phenotypic resistance was defined as this percentage.

\section{Genotype analysis}

DNA was extracted from 30 individuals per replicate using the salt extraction method [25], and then tested with allele-specific real time PCR to determine genotype at the 1534 and 1016 loci following protocols described in Alvarez et al. [26].

\section{Statistical analyses}

Allele frequencies for C1534 and I1016 were calculated for each population before and after the experiment. To test for linkage disequilibrium between the two markers, we calculated the coefficient $\mathrm{D}$ and the resulting $\mathrm{r}^{2}$ following the equations outlined in Gillespie [27] and used a Chi-square test with one degree of freedom to test the statistical significance. The change in genotype was analyzed with a Chi-square test of independence, and the difference between densities in the proportion knocked down with insecticide was assessed with a Welch t-test. Differences in development time between high and low density, defined as the total number of days from first-instar larva to adult, was quantified using a linear mixed effects model with replicate as a random intercept ( $\mathrm{R}$ package nlme [28]). The probability of survival was analyzed using a binomial-distributed generalized linear mixed effects model (GLMM), also with replicate as a random intercept ( $\mathrm{R}$ package lme4 [29]). Analyses were conducted with the $R$ statistical program version 3.3.2 [30].

\section{Aedes aegypti strain description}

Pyrethroid susceptible and resistant Ae. aegypti field strains were generated from eggs collected in the cities of Cienega de Flores (Nuevo Leon State, Mexico, susceptible strain) and Uman (Yucatan State, Mexico, resistant strain). First generation (F1) mosquito larvae were used for the experiments. Initial gene frequencies for each colony for the C1534 and I1016 kdr mutations were quantified using allele-specific real time PCR on 50 randomly selected F0 adult mosquitoes, applying the protocols described in Alvarez et al. [26]. The susceptible population had a $10 \%$ frequency of both $k d r$ mutations and $100 \%$ knockdown to permethrin at the diagnostic time, and the resistant population had a $98 \%$ frequency of the C1534 mutation, 73\% of the I1016 mutation and $13 \%$ knockdown to permethrin at the diagnostic time. Additional information on the resistant and susceptible strains can be found in Deming et al. [22] and Siller et al. [31], respectively. Rates of phenotypic resistance to the pyrethroid permethrin were estimated using the standardized CDC bottle bioassays following published guidelines on 100 F1 mosquitoes of each colony [24].

We acknowledge that our experimental strains are derived from different locations and therefore do not share the same genetic background, though we are only using the susceptible strain as a control. The statistical analysis conducted does not compare the strains; rather, it compares each strain to itself given density treatment (high and low) since that is our main question of interest. Including the susceptible strain allows us to rule out other environmental conditions that could be causing changes in resistance status.

\section{Results \\ Larval development}

The mean ( \pm standard deviation) number of days from first-instar larva to adult was $12.3 \pm 0.6$ days longer at high density than low density (Fig. 1a; GLMM generalized linear mixed-effects model, $t=21.93, d f=5856, P<0$. 0001). Similarly, the survival probability from first-instar larva to adult was significantly lower for individuals in the high density treatment than the low density (Fig. 1b; Binomial GLMM, odds ratio OR $=0.15$; 95\% CI: 0.11-0.20).

\section{Phenotypic resistance}

Expression of phenotypic resistance to the pyrethroid insecticide permethrin was significantly lower in the resistant population at high density compared to low density (Fig. 2; Welch t-test, $t=-3.41, d f=4.5, P=0.0225$ ). At 


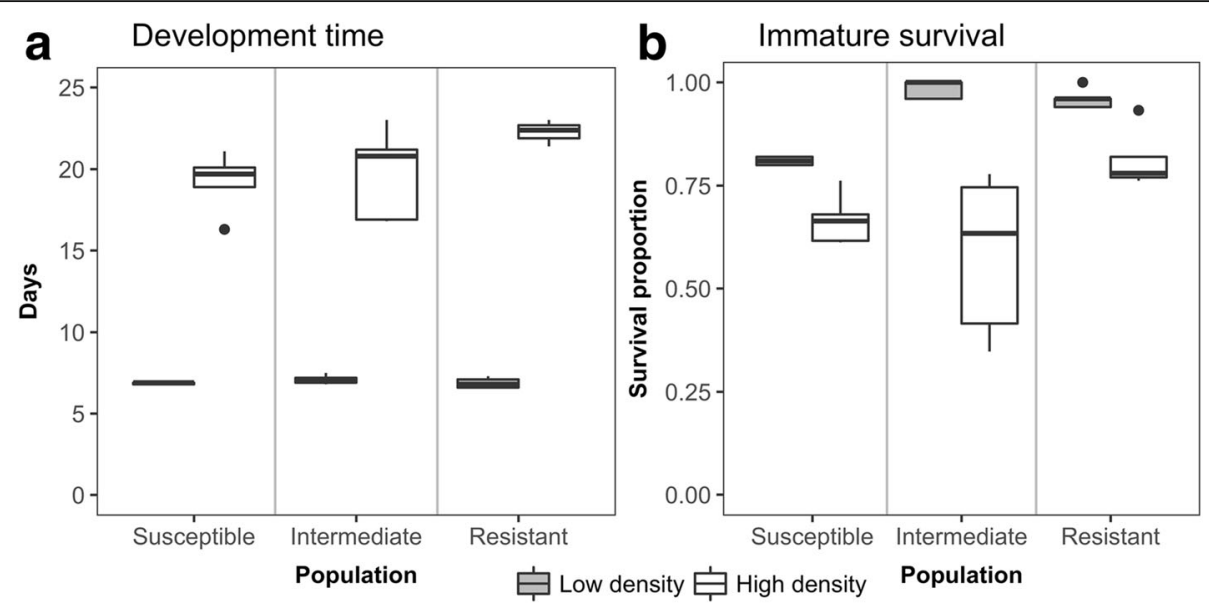

Fig. 1 Larval performance for each population and density. Boxplots show the distribution of a development time, defined as total time from first-instar to adult and $\mathbf{b}$ immature survival, measured as the proportion of the population surviving to adult of all five replicates

low density, only an average of $48.2 \pm 28.5 \%$ of the resistant population was knocked down at 30 minutes, but at high density, an average of $93 \pm 7.1 \%$ were knocked down. This significant reduction in phenotypic resistance at high density rendered the originally "resistant" population susceptible according to the WHO guidelines, which mark the resistance threshold at 90\% population knockdown at the diagnostic time for the CDC bioassays [32]. An increase in susceptibility at high density was also seen in the intermediate population, with an average $80.4 \pm 16.7 \%$ of the population knocked down at low density, yet an average of $97.8 \pm 2.0 \%$ knocked down at high density (Fig. 2).

\section{$k d r$ allele frequencies}

At both densities, the C1534 allele frequency of the resistant population was significantly reduced from a starting overall frequency of 0.98 to an average \pm SD

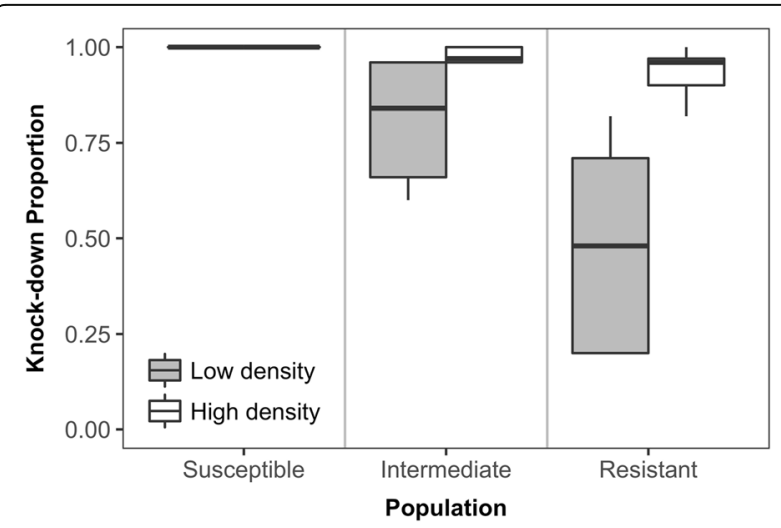

Fig. 2 Phenotypic resistance changes based on density conditions. Boxplots show the distribution of the proportion of the population knocked down in the presence of the diagnostic dose of permethrin according to CDC bottle bioassay standard procedures frequency of $0.93 \pm 0.05$ at low density (Fisher's exact test, $P=0.003$ ) and $0.69 \pm 0.04$ at high density (Fisher's exact test, $P<0.0001$ ) (Fig. 3). This marked effect of density on allele frequency change was not observed for the I1016 mutation (Fig. 3). The frequency of the I1016 allele increased slightly after both treatments, though not significantly (low density: Chi-square $=0.47, P=0$. 492; high density: Chi-square $=3.3, P=0.068$ ).

\section{Discussion}

We found that density-dependent intraspecific competition can act as a selective force to regain susceptibility in pyrethroid-resistant Ae. aegypti mosquitoes. High density larval conditions induced competition, evidenced through reduced immature survival and delayed development time. Consequently, this heightened competition selected for individuals without the $\mathrm{C} 1534 k d r$ mutation, causing a striking decrease in its frequency in the resistant population only after one generation of selection. Such rapid evolution gives insights into the maintenance of polymorphism at $k d r$ sites in Ae. aegypti field populations. Although insecticide selection pressures are strong, they are rarely uniform in time or space, as they are largely driven by disease outbreaks [33]. In the absence of insecticide, population densities may increase, imposing stronger competition and selection towards susceptibility. The alternation of insecticide selection pressure with selection due to density-dependence may, in part, account for the genetic variation at $k d r$ loci and can be leveraged to mitigate resistance evolution.

Equally important is that phenotypic susceptibility was re-established in the resistant population through a gene-environment interaction. Larvae from the same parent population with high resistance exhibited different resistance phenotypes depending on the conditions in which they were raised. If raised with minimum 


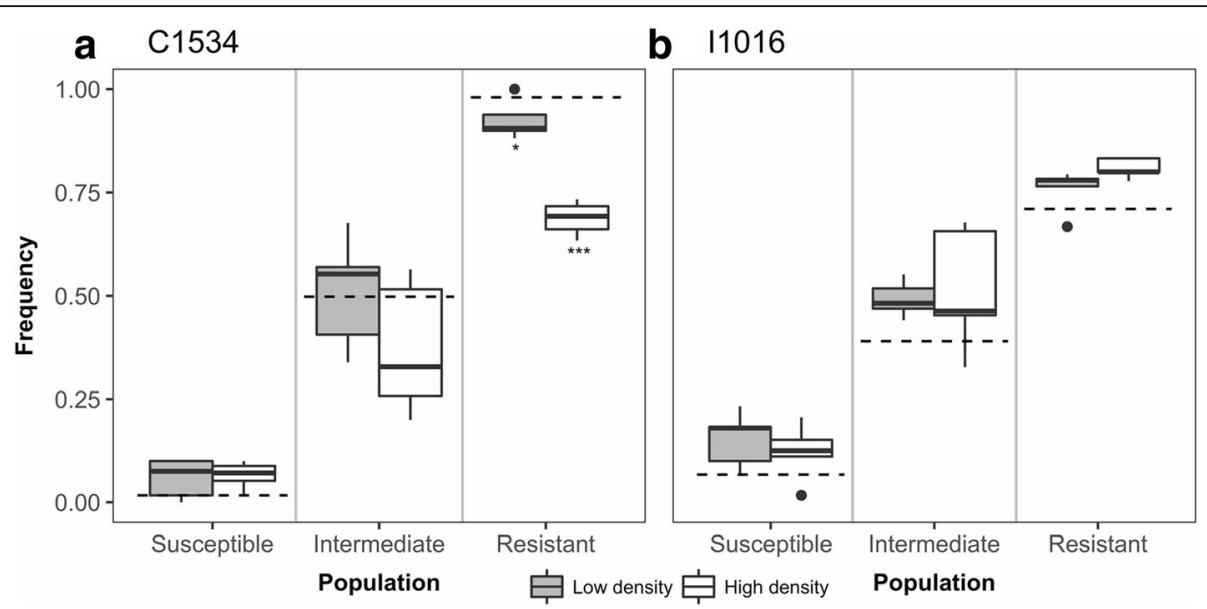

Fig. $3 \mathrm{kdr}$ frequencies as a result of density-dependent selection. Box plots show the distribution of the $k d r$ frequencies of adult mosquitoes emerging from each treatment combination. The dotted line represents the initial frequency of first-instar larvae in the population. ${ }^{*} P<0.01,{ }^{* * *} P$ $<0.0001$

intraspecific competition (low density), they remained resistant; however, if they were raised under strong competition (high density), they became diagnostically susceptible according to WHO guidelines [32]. Not only do these results demonstrate that phenotypes can be altered based on environmental conditions, but they also raise concerns about the external validity of biological assays used to phenotypically characterize the levels of resistance of natural populations. Based on our findings, we hypothesize that the standard resistance assays (CDC bottle bioassay and the WHO susceptibility test) would provide different results if performed with adults collected from the field (that are naturally constrained by food and density) versus adults reared in optimal laboratory conditions. This potential discrepancy could bias results from standard bioassay assays that are used to monitor resistance in field populations, providing incomplete or inaccurate information for vector control. Further evaluations of the bioassay methodology are needed, including the investigation of the correlation between phenotype and genotype at higher insecticide doses and the interplay between density and phenotypic resistance.

The impact of the larval environment on phenotypic resistance in adults has also been demonstrated in Anopheles spp. Owusu et al. [5] found that a nominally pyrethroid-resistant population of An. stephensi became susceptible according to WHO guidelines (using WHO tube tests) if reared with only $25 \%$ of the larval food available. Additionally, Oliver et al. [6] found that DDTresistant $A n$. arabiensis had decreased time to mortality using the bottle bioassay when larvae were on a restricted diet compared to well-fed larvae. Given these pervasive effects across species, it seems important to re-evaluate existing diagnostic assays for resistance as well as understand how resistance may change with varying environmental conditions.

This study investigated the effect of density-dependent competition on pyrethroid resistance in only one generation, though it would be beneficial to assess the impacts over multiple generations of density-induced selection. While we found a marked reduction in the C1534 $k d r$ mutation frequency in one generation, we did not find a significant change in the I1016 mutation. This is likely because we only conducted the experiment over one generation; when Brito et al. [12] reared a population of Ae. aegypti with the I1016 $k d r$ mutation in the absence of insecticide for 15 generations, they found a decrease in the I1016 allele from $50 \%$ to $21.7 \%$, which would on average produce a $1.8 \%$ change in one generation. Assessing the longer-term effects of density-induced selection could aid in our understanding of both field dynamics and potential fitness costs of the $k d r$ mutations.

\section{Conclusions}

Here we show adult pyrethroid resistance can be mediated by larval rearing density. Intraspecific competition induced by high densities can render a "resistant" population susceptible and act as a selective force to reduce the $\mathrm{C} 1534 k d r$ allele frequency. Given that Ae. aegypti are container breeders and subject to density-dependence in the field, the results give insight into one of the mechanisms driving insecticide resistance evolution in field populations. Furthermore, the results highlight the deficiencies in current diagnostic assays for resistance and suggest that future research should explore how to make these assays more robust to differences in environmental conditions. 


\section{Abbreviations}

CDC: Centers for Disease Control and Prevention;

DDT: Dichlorodiphenyltrichloroethane; kdr: knockdown resistance;

PCR: polymerase chain reaction; WHO: World Health Organization

\section{Acknowledgements}

We would like to thank Karina Villanueva (Universidad Autonoma de Nuevo Leon, Mexico) and Julian Rodriguez (Emory University, USA) for processing mosquitoes in the laboratory, Parker Cleveland for his help conducting the experiments, and Levi Morran and Joseph R. McMillan for providing helpful comments to earlier versions of this manuscript.

\section{Funding}

This project was funded by grants from National Science Foundation (NSF/DEB/DDIG \#1601520), and Mexico's CONACYT (Project \#255141 \& Project \#INFRA-251675)

\section{Availability of data and materials}

The datasets generated during the current study are available in the Dryad repository (accession: doi:10.5061/dryad.sc1156g).

\section{Authors' contributions}

MG and VUP performed experiments and analysis. MG, PMS, AEF and GVP wrote the manuscript. All authors read and approved the final manuscript.

\section{Ethics approval and consent to participate}

Not applicable.

\section{Competing interests}

The authors declare that they have no competing interests.

\section{Publisher's Note}

Springer Nature remains neutral with regard to jurisdictional claims in published maps and institutional affiliations.

\section{Author details}

'Department of Entomology, Pennsylvania State University, University Park, Pennsylvania, PA 16802, USA. ${ }^{2}$ Departamento de Zoología, Campus de Ciencias Biológicas y Agropecuarias, Universidad Autónoma de Yucatán, Apartado Postal 4-116, Itzimna, 97000 Mérida, Yucatan, Mexico. ${ }^{3}$ Universidad Autónoma de Nuevo León, Facultad de Ciencias Biológicas, Av. Universidad s/n Cd. Universitaria, San Nicolas de los Garza, 66455 Nuevo Leon, Mexico. ${ }^{4}$ Department of Environmental Sciences, Emory University, 400 Dowman Drive, Atlanta, GA 30322, USA.

Received: 11 January 2018 Accepted: 23 April 2018 Published online: 03 May 2018

\section{References}

1. Liu N. Insecticide resistance in mosquitoes: impact, mechanisms, and research directions. Annu Rev Entomol. 2015;60:537-59.

2. Hemingway J, Ranson H. Insecticide resistance in insect vectors of human disease. Annu Rev Entomol. 2000;45:371-91.

3. REX Consortium. Heterogeneity of selection and the evolution of resistance. Trends Ecol Evol. 2013:28:110-8.

4. Du Y, Nomura Y, Zhorov BS, Dong K. Sodium channel mutations and pyrethroid resistance in Aedes aegypti. Insects. 2016;7:1-11.

5. Owusu HF, Chitnis N, Müller P. Insecticide susceptibility of Anopheles mosquitoes changes in response to variations in the larval environment. Sci Rep. 2017;7:1-9

6. Oliver SV, Brooke BD. The effect of larval nutritional deprivation on the life history and DDT resistance phenotype in laboratory strains of the malaria vector Anopheles arabiensis. Malar J. 2013;12:44

7. Glunt KD, Paaijmans KP, Read AF, Thomas MB. Environmental temperatures significantly change the impact of insecticides measured using WHOPES protocols. Malar J. 2014:13:350.

8. Gubler DJ. Epidemic dengue/dengue hemorrhagic fever as a public health, social and economic problem in the 21st century. Trends Microbiol. 2002;10: 100-3.

9. Guzman MG, Halstead SB, Artsob H, Buchy P, Farrar J, Gubler DJ, et al. Dengue: a continuing global threat. Nat Rev Microbiol. 2010;8:S7-16.
10. Vontas J, Kioulos E, Pavlidi N, Morou E, della Torre A, Ranson H. Insecticide resistance in the major dengue vectors Aedes albopictus and Aedes aegypti. Pestic Biochem Physiol. 2012;104:126-31.

11. Soderlund DM, Knipple DC. The molecular biology of knockdown resistance to pyrethroid insecticides. Insect Biochem Mol Biol. 2003;33:563-77.

12. Brito LP, Linss JG, Lima-Camara TN, Belinato TA, Peixoto AA, Lima JB, et al. Assessing the effects of Aedes aegypti kdr mutations on pyrethroid resistance and its fitness cost. PLoS One. 2013;8:e60878.

13. Martins AJ, Ribeiro CD, Bellinato DF, Peixoto AA, Valle D, Lima JB. Effect of insecticide resistance on development, longevity and reproduction of field or laboratory selected Aedes aegypti populations. PLoS One. 2012;7:e31889.

14. Alvarez-Gonzalez LC, Briceño A, Ponce-Garcia G, Villanueva-Segura OK, Davila-Barboza JA, Lopez-Monroy B, et al. Assessing the effect of selection with deltamethrin on biological parameters and detoxifying enzymes in Aedes aegypti (L.). Pest Manag Sci. 2017:73:2287-93.

15. Walsh RK, Facchinelli L, Ramsey JM, Bond JG, Gould F. Assessing the impact of density dependence in field populations of Aedes aegypti. J Vector Ecol. 2011;36:300-7.

16. Alto BW, Bettinardi DJ, Ortiz S. Interspecific larval competition differentially impacts adult survival in dengue vectors. J Med Entomol. 2015;52:163-70.

17. Reiskind $\mathrm{MH}$, Lounibos LP. Effects of intraspecific larval competition on adult longevity in the mosquitoes Aedes aegypti and Aedes albopictus. Med Vet Entomol. 2009;23:62-8

18. Legros M, Lloyd AL, Huang Y, Gould F. Density-dependent intraspecific competition in the larval stage of Aedes aegypti (Diptera: Culicidae): revisiting the current paradigm. J Med Entomol. 2009:46:409-19.

19. Christophers SSR. Aedes aegypti (L.) The Yellow Fever Mosquito: Its life history, bionomics and structure. New York: Cambridge University Press; 1960.

20. Arrivillaga J, Barrera R. Food as a limiting factor for Aedes aegypti in waterstorage containers. J Vector Ecol. 2004;29:11-20.

21. Raymond B, Sayyed AH, Wright DJ. Genes and environment interact to determine the fitness costs of resistance to Bacillus thuringiensis. Proc R Soc London Ser B Biol Sci. 2005:272:1519-24.

22. Deming R, Manrique-Saide P, Medina Barreiro A, Cardeña EUK, CheMendoza A, Jones B, et al. Spatial variation of insecticide resistance in the dengue vector Aedes aegypti presents unique vector control challenges. Parasit Vectors. 2016:9:67

23. Manrique-Saide P, Coleman P, McCall P, Lenhart A, Vazquez-Prokopec GM, Davies C. Multi-scale analysis of the associations among egg, larval and pupal surveys and the presence and abundance of adult female Aedes aegypti (Stegomyia aegypti) in the city of Merida, Mexico. Med Vet Entomol. 2014:28:264-72.

24. Centers for Disease Control and Prevention. Guideline for evaluating insecticide resistance in vectors using the CDC bottle bioassay. Atlanta, GA: Centers for Disease Control and Prevention. https://www.cdc.gov/malaria/ resources/pdf/fsp/ir manual/ir_cdc bioassay en.pdf.

25. Black W, DuTeau N. RAPD PCR and SSCP analysis for insect population genetic studies. In: Crampton J, Beard CB, Louis C, editors. The molecular biology of insect disease vectors: a methods manual. New York: Chapman and Hall; 1997. p. 361-73.

26. Alvarez LC, Ponce G, Saavedra-Rodriguez K, Lopez B, Flores AE. Frequency of V1016I and F1534C mutations in the voltage-gated sodium channel gene in Aedes aegypti in Venezuela. Pest Manag Sci. 2015;71:863-9.

27. Gillespie J. Population genetics. 2nd ed. Baltimore: Johns Hopkins University; 2004

28. Pinheiro J, Bates D, DebRoy S, Sarkar D, R Core Team. nlme: linear and nonlinear mixed effects models. Vienna: R Foundation for Statistical Computing; 2016.

29. Bates D, Maechler M, Bolker BM, Walker S. Fitting linear mixed-effects models using Ime4. J Stat Softw. 2015;67:1-48.

30. R Core Team. R: a language and environment for statistical computing. Vienna: R Foundation for Statistical Computing; 2015.

31. Siller Q, Ponce G, Lozano S, Flores AE. Update on the frequency of Ile1016 mutation in voltage-gated sodium channel gene of Aedes aegypti in Mexico. J Am Mosq Control Assoc. 2011;27:357-62.

32. World Health Organization Global Malaria Programme. Test procedures for insecticide resistance monitoring in malaria vector mosquitoes. 2nd ed. Geneva: WHO; 2016.

33. Bowman LR, Donegan S, McCall PJ. Is dengue vector control deficient in effectiveness or evidence?: Systematic review and meta-analysis. PLoS Negl Trop Dis. 2016;10:3. 